\title{
Gadolinium as an accelerator for reaching thermal equilibrium and its influence on the ground state of $\mathrm{Dy}_{2} \mathrm{GdN} @ \mathrm{C}_{80}$ single-molecule magnets
}

\author{
Aram Kostanyan, ${ }^{1,2}$ Christin Schlesier, ${ }^{3}$ Rasmus Westerström,,${ }^{4,5}$ Jan Dreiser, ${ }^{2}$ Fabian Fritz, ${ }^{6}$ Bernd Büchner, ${ }^{3}$ \\ Alexey A. Popov, ${ }^{3}$ Cinthia Piamonteze, ${ }^{2}$ and Thomas Greber $\oplus^{1, *}$ \\ ${ }^{1}$ Physik-Institut, Universität Zürich, Winterthurerstrasse 190, CH-8057 Zürich, Switzerland \\ ${ }^{2}$ Swiss Light Source, Paul Scherrer Institut, CH-5232 Villigen PSI, Switzerland \\ ${ }^{3}$ Leibniz Institute of Solid State and Materials Research, Dresden, D-01069 Dresden, Germany \\ ${ }^{4}$ Division of Synchrotron Radiation Research, Institute of Physics, SE-221 00 Lund, Sweden \\ ${ }^{5}$ NanoLund, Lund University, Box 118, 22100 Lund, Sweden \\ ${ }^{6}$ Department of Physics, University Osnabrück, D-49076 Osnabrück, Germany
}

(Received 21 June 2020; revised 25 November 2020; accepted 7 December 2020; published 5 January 2021)

\begin{abstract}
Endohedral fullerenes are perfect nanolaboratories for the study of magnetism. The substitution of a diamagnetic scandium atom in $\mathrm{Dy}_{2} \mathrm{ScN} @ \mathrm{C}_{80}$ with gadolinium decreases the stability of a given magnetization and demonstrates $\mathrm{Gd}$ to act as a single atom catalyst that accelerates the reaching of thermal equilibrium. X-ray magnetic circular dichroism at the $M_{4,5}$ edges of Gd and Dy shows that Gd affects the ground state. The Gd magnetic moment follows the sum of the external and the dipolar magnetic field of the two Dy ions and compared to $\mathrm{Dy}_{2} \mathrm{ScN} @ \mathrm{C}_{80}$ a lower exchange barrier is found between the ferromagnetic and the antiferromagnetic Dy configuration. The Arrhenius equilibration barrier as obtained from superconducting quantum interference device magnetometry is more than one order of magnitude larger, though a much smaller prefactor imposes the faster equilibration in $\mathrm{Dy}_{2} \mathrm{GdN} @ \mathrm{C}_{80}$. This sheds light on the importance of angular momentum balance and symmetry in magnetic relaxation.
\end{abstract}

DOI: 10.1103/PhysRevB.103.014404

\section{INTRODUCTION}

A catalyst accelerates the approach of thermal equilibrium. In the classical picture it lowers the kinetic barrier between two states $A$ and $B$ where the transition rates are described by Boltzmann factors comprising the barrier, the energy difference between $A$ and $B$ and by a prefactor reminiscent to an attempt frequency. The concept of a catalyst can as well be applied for the case of single molecule magnets (SMMs) that were prepared in a state outside thermal equilibrium towards which they decay with certain rates. From the temperature dependence of these rates Arrhenius barriers and attempt frequencies may be inferred. The exponential prefactors in the kinetics reflect the energy landscape where a process runs. In the present case the $4 f$ electron spins of a single gadolinium atom are shown to catalyze magnetic transition rates of the $\mathrm{Dy}_{2}$ dimer in $\mathrm{Dy}_{2} \mathrm{GdN} @ \mathrm{C}_{80}$ dramatically, which is not only due to the fact that $\mathrm{Gd}$ affects the ground state energies of the molecule.

SMMs display hysteresis, i.e., maintain nonequilibrium magnetization for a measurably long period of time [1-3]. After the discovery of single-ion molecule magnets [4] the lanthanide magnetochemistry got a significant boost where new strategies for the improvement were developed [5-7]. With these ideas on rational design magnetic hysteresis of

*greber@physik.uzh.ch single molecules at $60 \mathrm{~K} \mathrm{[8]} \mathrm{and} \mathrm{even} \mathrm{above} \mathrm{liquid-nitrogen}$ temperatures [9] is reached by now. Endohedral fullerenes provide a versatile environment to encapsulate different diamagnetic and paramagnetic ions [10,11]. First magnetic studies were performed on $\mathrm{Gd} @ \mathrm{C}_{82}$ [12], and it took 17 more years until SMM behavior in endohedral fullerenes was found with $\mathrm{DySc}_{2} \mathrm{~N} @ \mathrm{C}_{80}$ [13]. In the following many different $\mathrm{C}_{80}$ mixed dysprosium-lanthanide nitride clusters have been synthesized and magnetically characterized [14-16]. As in other radical bridged lanthanide complexes [17] dysprosium ion pairs appear to form excellent SMMs [18]. For the case of $\mathrm{Dy}_{2} \mathrm{ScN} @ \mathrm{C}_{80}$ the particularly long zero-field lifetime of the magnetization was attributed to exchange protection of two Kramers ions [19]. Heterometallic clusterfullerenes require more effort for synthesis and separation, and only recently an endeavor of implementing three different rare-earth atoms [20]. If more than one paramagnetic atomic species is involved, element specific methods, such as X-ray magnetic circular dichroism (XMCD) [21] give unprecedented insight into the magnetic ordering of heteroatomic clusters [22-24].

Here we investigate the interplay between two Dy and one Gd ion in a 1-nm carbon cage. The substitution of scandium with gadolinium decreases the excitation gap between the ferro- and antiferromagnetically coupled Dy doublets. On the other hand, the kinetic barrier increases but the prefactor still dictates a lower magnetization lifetime in $\mathrm{Dy}_{2} \mathrm{GdN} @ \mathrm{C}_{80}$. This strong change in magnetization dynamics indicates $\mathrm{Gd}$ to break the spin-flip protection symmetry which is found in 
$\mathrm{Dy}_{2} \mathrm{ScN} @ \mathrm{C}_{80}$ and exhibits the role of the Gd angular momentum in the change of magnetization.

\section{EXPERIMENTAL METHODS}

$\mathrm{Dy}_{2} \mathrm{GdN} @ \mathrm{C}_{80}$ endofullerenes were produced by arcdischarge synthesis using the corresponding metals [15]. The subsequent separation by high-pressure liquid chromatography resulted in a sample of $95 \%$ purity with $5 \% \mathrm{Dy}_{3} \mathrm{~N} @ \mathrm{C}_{80}$ contamination as inferred from time-of-flight mass spectrometry [25]. The $\mathrm{Dy}_{3} \mathrm{~N} @ \mathrm{C}_{80}$ content does not affect the conclusions of this paper. The magnetization measurements were performed by XMCD and superconducting quantum interference device (SQUID) magnetometry. XMCD was performed at the X-Treme beamline of the Swiss light source [26] on a sample obtained after spray coating of a toluene solution of the molecules on an aluminum substrate. The total electron yield was normalized by the secondary electron current from a gold mesh in the x-ray beam before it hits the sample and by the X-ray absorption cross section of gold. The SQUID magnetometer was a Quantum Design (QD) MPMS3 vibrating sample magnetometer where we performed magnetization experiments in external magnetic fields up to $7 \mathrm{~T}$ and temperatures down to $1.55 \mathrm{~K}$. For the SQUID measurements, the toluene solution was dropcast into a QD polypropylene powder sample holder. A temperature-independent diamagnetic background of $-5.8 \times 10^{-8} \mathrm{Am}^{2} / \mathrm{T}$ was inferred from temperature-dependent magnetization measurements. From the paramagnetic saturation at $2 \mathrm{~K}$ and $7 \mathrm{~T}, m_{\mathrm{sat}}$ of $2.17 \times$ $10^{-6} \mathrm{Am}^{2}$ and a maximum magnetic moment of $17 \mu_{B}$ an ensemble of $1.4 \times 10^{16}$ molecules with a mass of $33 \mu \mathrm{g}$ is inferred. (The saturation magnetic moment of $17 \mu_{B}$ being the sum of two noncollinear Dy $J_{z}=15 / 2$ and one collinear Gd $S=7 / 2$ moment.)

\section{RESULTS AND DISCUSSION}

\section{A. Ground state}

Figure 1 shows the ground-state model for $\mathrm{Dy}_{2} \mathrm{GdN} @ \mathrm{C}_{80}$ at zero external magnetic field. The electric field of the nitrogen ion lifts the 16-fold degeneracy of the Dy ${ }^{6} \mathrm{H}_{15 / 2}$ Hund ground state $[19,27]$, whereas no ligand field splitting is expected for the half-filled $4 f$ shell of the $\mathrm{Gd}^{8} \mathrm{~S}_{7 / 2}$ configuration. Since the ligand field interaction is much stronger than mutual magnetic interaction, it is expected that the Dy ions assume as in $\mathrm{Dy}_{2} \mathrm{ScN} @ \mathrm{C}_{80}, J_{z}=15 / 2$ states. At low temperatures the higher-lying states in the $J_{z}$ manifold of dysprosium may be neglected, and the picture of two pseudospins applies [19]. In zero field, the two pseudospins of the Dy ions arrange in two time-reversal symmetric doublets $|1\rangle,|\overline{1}\rangle$ (ferromagnetic), and $|2\rangle,|\overline{2}\rangle$ (antiferromagnetic). The two Dy atoms in the ferromagnetic configuration create at the Gd site a dipole field of $180 \mathrm{mT}$ parallel to the Dy-Dy axis, whereas in the antiferromagnetic configuration it is $720 \mathrm{mT}$ and directed perpendicular to the Dy-Dy axis.

The ground state as proposed in Fig. 1 is confirmed by $\mathrm{X}$-ray magnetic circular dichroism. The XMCD for Dy and $\mathrm{Gd}$ is shown in the bottom of Fig. 1(a). Sum rule analysis [28-30] reveals effective saturation magnetic moments of $\mathrm{Gd}^{3+}$ and $\mathrm{Dy}^{3+}$ in $\mathrm{Dy}_{2} \mathrm{GdN} @ \mathrm{C}_{80}$ [25]. The Dy effective (a)

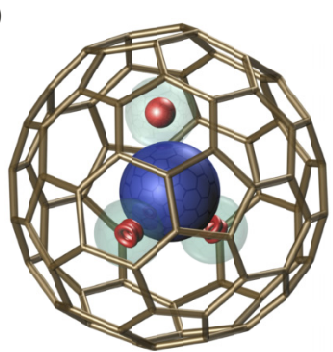

(c)

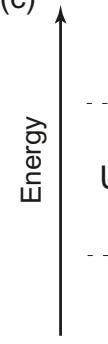

(b)

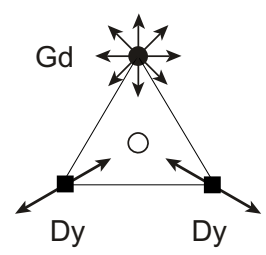

(d)

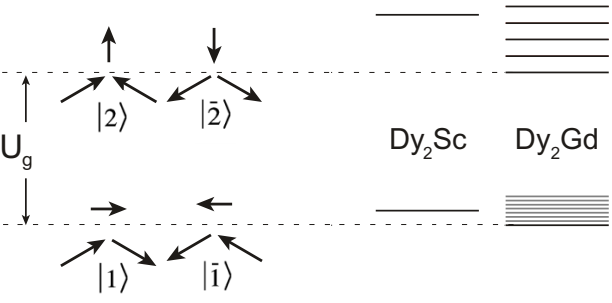

FIG. 1. (a) Ball and stick model of $\mathrm{Dy}_{2} \mathrm{GdN} @ \mathrm{C}_{80}$ with a van der Waals diameter of about $1.1 \mathrm{~nm}$. The endohedral ions are mimicked with their ion radii. In the center of the lanthanides (turquois) the 4f $J_{z}=15 / 2$ and $J_{z}=7 / 2$ orbitals for Dy and Gd are depicted in red. (b) Sketch of the endohedral unit with the orientations of the magnetic moments. The two-way arrows indicate the possible orientations of the $\mathrm{Dy}^{3+}$ moments that are aligned along the N-Dy axes, whereas the Gd moment is oriented along the total magnetic field. (c) Ground-state magnetic structure at zero external magnetic field as an extension of the pseudospin model for $\mathrm{Dy}_{2} \mathrm{ScN} @ \mathrm{C}_{80}$ [19]. The orientation of the $\mathrm{Gd}^{3+}$ moment is assumed by the $B$ field of the two Dy moments. $U_{g}$ is the energy difference between the lowest ferromagnetic $(|1\rangle,|\overline{1}\rangle)$ and the lowest antiferromagnetic states $(|2\rangle,|\overline{2}\rangle)$. (d) Energy levels of $\mathrm{Dy}_{2} \mathrm{ScN} @ \mathrm{C}_{80}$ and $\mathrm{Dy}_{2} \mathrm{GdN} @ \mathrm{C}_{80}$. The eight electron spin states of Gd are split by the $B$ field of the Dy ions. Note: Here the average ferro- antiferrosplittings are assumed to be equal for both molecules, which turns out not to be the case.

magnetic moment at saturation of $4.5 \pm 0.1 \mu_{B}$ compares well with the value in $\mathrm{DySc}_{2} \mathrm{~N} @ \mathrm{C}_{80}$ [13]. The moment of $\mathrm{Gd}$ gets $5.8 \pm 0.1 \mu_{B}$. It is smaller than the expectation for a free collinear $\mathrm{Gd}$ ion as also observed in other Gd molecular magnet systems [31].

The magnetization curves of $\mathrm{Gd}$ and Dy are shown in Fig. 2(b). For endohedral Gd it deviates from a Brillouin function with $g=2$ and $S=7 / 2$. This certifies endohedral Gd not to behave as a free ion but that it is subject to magnetic interaction with the two Dy ions. Compared to the free ion, the relative magnetization of $\mathrm{Gd}$ in $\mathrm{Dy}_{2} \mathrm{GdN} @ \mathrm{C}_{80}$ is largest at a field of about $0.2 \mathrm{~T}$, which is close to the field imposed by the two Dy ions in the ferro ground states.

The above statements are substantiated with the implementation of a pseudospin model to the level scheme in Fig. 1(c) and 1(d) [25]. The solid lines in Fig. 2(b) are the best fit where access to the individual magnetization curves of Dy and Gd improves the reliability of the extracted parameters significantly. The magnetic moment of Dy gets $8.8 \mu_{B}$ and is in line with that in $\mathrm{Dy}_{2} \mathrm{ScN} @ \mathrm{C}_{80}[19]$, whereas that of $\mathrm{Gd}$ fits best to the value of $6.9 \mu_{B}$. The parameter $B_{D}$ [25] that describes the splitting of the Gd states due to the Dy dipolar fields is $0.24 \mathrm{~T}$, 

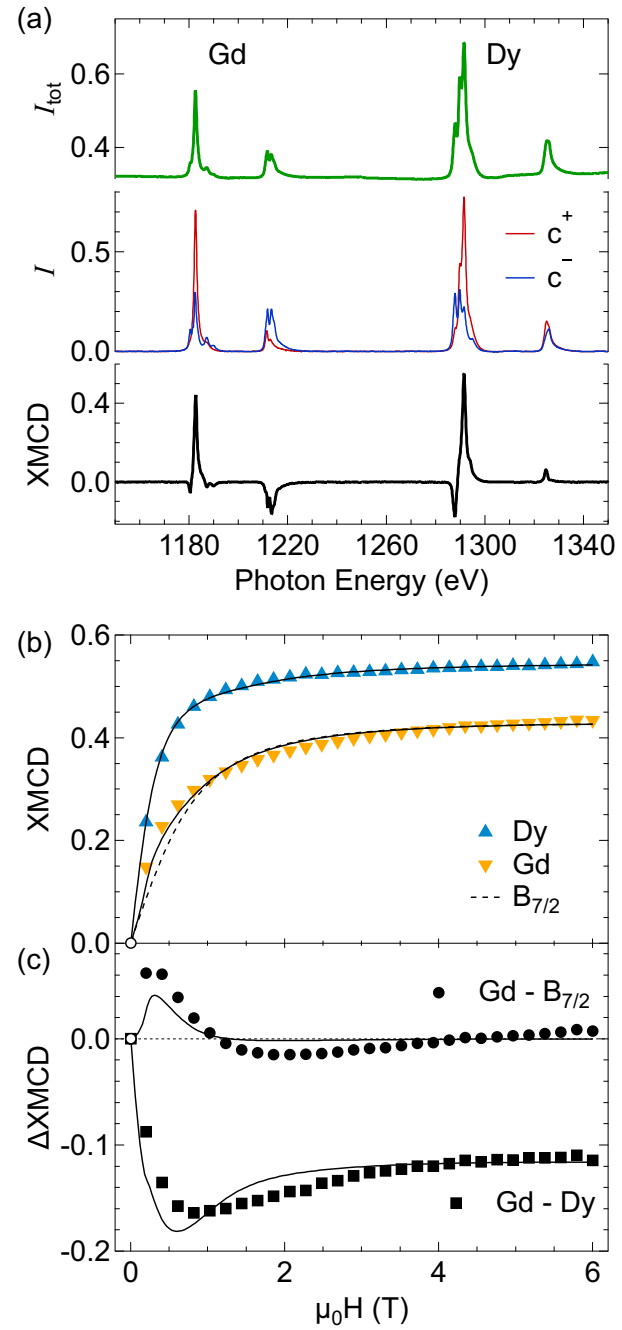

FIG. 2. (a) Top: X-ray absorption spectrum $I_{\text {tot }}$ vs. photon energy of $\mathrm{Dy}_{2} \mathrm{GdN} @ \mathrm{C}_{80}$ (green line). The intensity ratio between Gd and Dy is in line with the 1:2 stoichiometry. Maximum electron yield 106 pA. Middle: Background subtracted x-ray absorption spectra $I\left(c^{+}\right)$and $I\left(c^{-}\right)$for both x-ray helicities with the maxima of the $\mathrm{Gd}$ and Dy intensities normalized to 1. External field 6.5 T, parallel to the $\mathrm{X}$-ray incidence. Bottom: XMCD $I\left(c^{+}\right)-I\left(c^{-}\right)$. (b) Field-dependent $\mathrm{XMCD}$ of Dy (up-triangle, light blue) and Gd (down-triangle, yellow). Brillouin function $B_{7 / 2}$ (dashed line) corresponding to $g=$ $2, J=7 / 2, T=1.95 \mathrm{~K}$, representing the magnetization of free $\mathrm{Gd}$. The solid lines are fits of the element specific magnetization curves to an extended pseudospin model [25]. (c) Differences between $\mathrm{XMCD}(\mathrm{Gd})$ and $B_{7 / 2}$ (black disks), and $\mathrm{XMCD}$ (Dy) (black squares). The solid lines are obtained from the fit results in (b). The peak of $\mathrm{XMCD}(\mathrm{Gd})-B_{7 / 2}$ indicates the $B$ field of the Dy ions at the Gd site.

which is close to the value of $0.2 \mathrm{~T}$ of a moment of $10 \mu_{B}$ at a Dy-Gd distance of $0.36 \mathrm{~nm}$. This affirms that in zero field, the Gd magnetism is governed by the dipolar fields of the two Dy ions and that possible exchange interaction between Dy and Gd must be much smaller. Finally, the excitation energy or gap between the lowest ferromagnetically and the lowest antiferromagnetically coupled states $U_{g}$ is determined from comparison of the data to the model. In $\mathrm{Dy}_{2} \mathrm{GdN} @ \mathrm{C}_{80}$ it decreases, compared to $\mathrm{Dy}_{2} \mathrm{ScN} @ \mathrm{C}_{80}$ from 9 to $0.1 \pm 0.8 k_{B} \mathrm{~K}$.
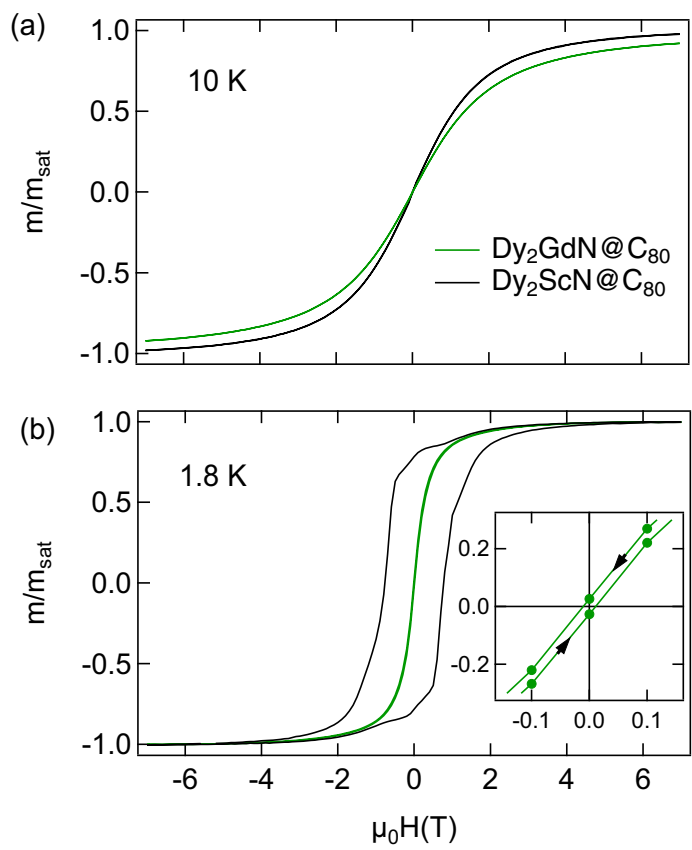

FIG. 3. Comparison of the magnetization of $\mathrm{Dy}_{2} \mathrm{GdN} @ \mathrm{C}_{80}$ (green) and $\mathrm{Dy}_{2} \mathrm{ScN} @ \mathrm{C}_{80}$ (gray) normalized with the corresponding saturation values $m_{\text {sat }}=m(7 \mathrm{~T}, 2 \mathrm{~K})$. (a) At $10 \mathrm{~K}$ both molecules show paramagnetic behavior where the initial slope of the normalized magnetization of $\mathrm{Dy}_{2} \mathrm{GdN} @ \mathrm{C}_{80}$ is a factor of 0.8 smaller than that of $\mathrm{Dy}_{2} \mathrm{ScN} @ \mathrm{C}_{80}$. (b) At $1.8 \mathrm{~K}$ with a field scan rate of $5.3 \mathrm{mT} / \mathrm{s}$ $\mathrm{Dy}_{2} \mathrm{GdN} @ \mathrm{C}_{80}$ shows a small hysteresis of $68 \mathrm{mT} m_{\text {sat }}$, whereas $\mathrm{Dy}_{2} \mathrm{ScN} @ \mathrm{C}_{80}$ displays a large hysteresis with an area of $3190 \mathrm{mT}$ $m_{\text {sat }}$. The inset zooms the hysteresis of $\mathrm{Dy}_{2} \mathrm{GdN} @ \mathrm{C}_{80}$ with the axes $m / m_{\text {sat }}$ and $\mu_{0} \mathrm{H}(\mathrm{T})$. Black arrows indicate the field scan directions.

This $U_{g}$ value points to zero-field degeneracy of the four possible Dy spin configurations. It is only partly due to the higher Dy-Gd coupling in the antiferromagnetic states, which accounts to $2.5 k_{B} \mathrm{~K}$, but is also due to a smaller Dy-Dy exchange for the case of $\mathrm{Dy}_{2} \mathrm{GdN} @ \mathrm{C}_{80}$. Such variations in exchange coupling are difficult to predict but were even observed between $\mathrm{Dy}_{2} \mathrm{ScN} @ \mathrm{C}_{80}$ and $\mathrm{Dy}_{2} \mathrm{LuN} @ \mathrm{C}_{80}$, where both scandium and lutetium are diamagnetic [16].

\section{B. Catalytic action of $\mathbf{G d}$}

The smaller barrier $U_{g}$ between the ferro- and the antiferrostates is a hint that $\mathrm{Gd}$ may accelerate the approach of the Dy spin system to thermal equilibrium. In Fig. 3 magnetization curves for the two molecules are displayed. At $10 \mathrm{~K}$ temperature both display paramagnetism with a characteristic thermal equilibrium curve. The relative zero-field susceptibility of $\mathrm{Dy}_{2} \mathrm{GdN} @ \mathrm{C}_{80}$ is a factor of 0.81 smaller than for $\mathrm{Dy}_{2} \mathrm{ScN} @ \mathrm{C}_{80}$. This confirms that more magnetic states are available in $\mathrm{Dy}_{2} \mathrm{GdN} @ \mathrm{C}_{80}$. The additional states of the $\mathrm{Gd}$ ion interfere with the $\mathrm{Dy}_{2}$ units and decrease the hysteresis of $\mathrm{Dy}_{2} \mathrm{ScN} @ \mathrm{C}_{80}$ at $1.8 \mathrm{~K}$ by a factor of 47 [see Fig. 3(b) and Supplemental Material [25]). Apparently, the exchange protection as it is operational in $\mathrm{Dy}_{2} \mathrm{ScN} @ \mathrm{C}_{80}$ breaks down if gadolinium sits in the same cage instead of scandium. This makes Gd an option in engineering of single molecule 

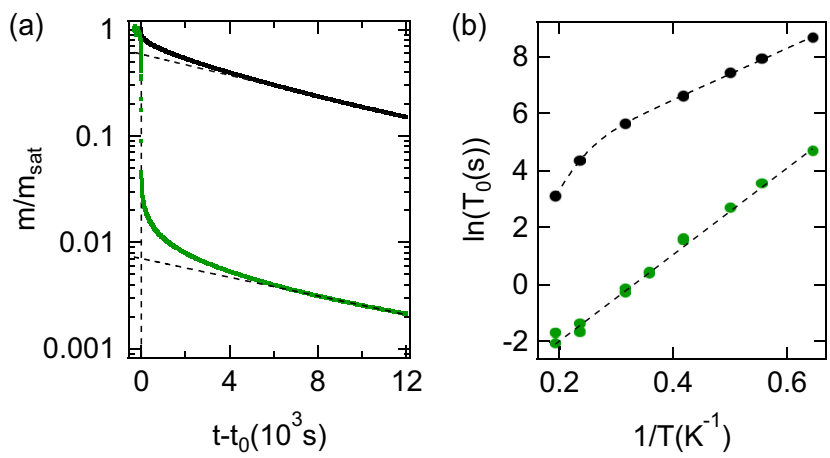

FIG. 4. Magnetization decay of $\mathrm{Dy}_{2} \mathrm{ScN} @ \mathrm{C}_{80}$ (black) and $\mathrm{Dy}_{2} \mathrm{GdN} @ \mathrm{C}_{80}$ (green) after saturating the samples at $7 \mathrm{~T}$ and ramping the magnet down to zero field in $350 \mathrm{~s}$. (a) Magnetization as a function of time at $1.55 \mathrm{~K} . t_{0}$ is the time when zero field is reached. (b) Arrhenius plot of the remanence times $\ln \left(T_{0}\right)$ for the two molecules at temperatures between 1.55 and $5 \mathrm{~K}$. For $\mathrm{Dy}_{2} \mathrm{ScN} @ \mathrm{C}_{80}$ (black) a low-temperature barrier of $9.0 \pm 0.2 \mathrm{~K}$ is found [19]. For $\mathrm{Dy}_{2} \mathrm{GdN} @ \mathrm{C}_{80}$ (green) a straight line leads $15.1 \pm 0.4 \mathrm{~K}$ with a $1 / 2500$ times smaller prefactor.

magnets, if, e.g., heat dissipation shall be minimized in highfrequency applications.

For all endofullerenes investigated so far, the ground-state parameter $U_{g}$ of the molecules as determined from the magnetization in thermal equilibrium was in line with the Arrhenius barrier $\Delta_{\text {eff }}$ from the decay of the magnetization [19,32]. In the following we will see that this is not the case for Dy ${ }_{2} \mathrm{GdN} @ \mathrm{C}_{80}$, and the discrepancy between $U_{g}$ and $\Delta_{\text {eff }}$ sheds light on the demagnetization dynamics.

The fluctuation rates of the magnetic states are expressed in the kinetics that describe the approach to thermal equilibrium. Figure 4(a) shows an experiment where the magnetic moments of the molecules were saturated in a magentic field of $7 \mathrm{~T}$, which is then ramped down to zero. The subsequent decay contains information on the ground state and the dynamics of the spin flips involved. The decay rate is not constant but decreases to a constant value. This behavior is common to single molecule magnets $[13,17]$. At $1.55 \mathrm{~K}$ and after $2 \mathrm{~h}$ the decay rates of $\mathrm{Dy}_{2} \mathrm{ScN} @ \mathrm{C}_{80}$ and $\mathrm{Dy}_{2} \mathrm{GdN} @ \mathrm{C}_{80}$ in the order of $10^{-4} \mathrm{~s}^{-1}$, although the backextrapolation of these rates to $t=t_{0}$ indicates that $60 \%$ of all $\mathrm{Dy}_{2} \mathrm{ScN} @ \mathrm{C}_{80} \mathrm{de}$ cayed with this rate, whereas it was only $0.7 \%$ in the case of $\mathrm{Dy}_{2} \mathrm{GdN} @ \mathrm{C}_{80}$. For a quantification of the kinetics that describe the approach to thermal equilibrium we investigate the decay rates as a function of temperature. It is, however, difficult to determine decay rates below $10^{-3} m_{\text {sat }}$ because of the relaxation of the magnet in the SQUID. In order to get a reliable value for the magnetic lifetime we evaluated the integral of the magnetization after reaching zero field at time $t_{0}$ that we like to call remanence time $T_{0}$,

$$
T_{0}=\int_{t_{0}}^{\infty}\left(m / m_{\mathrm{sat}}\right) d t
$$

where $T_{0}$ corresponds to the decay time $\tau$ of a single exponential $m\left(t-t_{0}\right)=m_{\text {sat }} \exp \left[-\left(t-t_{0}\right) / \tau\right]$. The validity of using this remanence time to describe the decay of the magnetization is justified by the evaluation of the $\mathrm{Dy}_{2} \mathrm{ScN} @ \mathrm{C}_{80}$ data [see Fig. 4(b)] where a barrier $\Delta_{\text {eff }} / k_{B}$ of $9.0 \pm 0.2 \mathrm{~K}$ is found as compared to $8.5 \pm 0.5 \mathrm{~K}$ with the standard method $[19,25]$. This barrier fits the dipole and exchange splitting in $\mathrm{Dy}_{2} \mathrm{ScN} @ \mathrm{C}_{80}$ as inferred from the equilibrium magnetization [19]. The Arrhenius slopes in Fig. 4(b) indicate for $\mathrm{Dy}_{2} \mathrm{GdN} @ \mathrm{C}_{80}$ a larger barrier than for $\mathrm{Dy}_{2} \mathrm{ScN} @ \mathrm{C}_{80}$. The barrier $\Delta_{\text {eff }}$ of $15.1 \pm 0.4 \mathrm{~K}$ cannot be understood with a decay path where the lowest ferrostates are directly excited into the lowest antiferrostates since $U_{g}$ is much lower. This suggests that the flip of the magnetization in a $|1\rangle \rightarrow|\overline{1}\rangle$ transition involves excitation of the Gd $4 f$ states. The high barrier is consistent with a picture where the Dy angular momentum change in a $|1\rangle \rightarrow|\overline{1}\rangle$ transition can be temporarily stored in the Gd ions. Furthermore, the kinetics of the magnetization decay comprises a prefactor $\tau_{0}$ in $\tau(T)=\tau_{0} \exp \left(\Delta_{\text {eff }} / k_{B} T\right)$. $\tau_{0}$ is a factor of $4.1 \pm 0.8 \times 10^{-4}$ smaller in $\mathrm{Dy}_{2} \mathrm{GdN} @ \mathrm{C}_{80}$ as compared to $\mathrm{Dy}_{2} \mathrm{ScN} @ \mathrm{C}_{80}$. It includes besides an attempt frequency as well the quantum symmetry of the object [32]. Apparently, single Gd atoms break the symmetry that imposes the exchange protection in $\mathrm{Dy}_{2} \mathrm{ScN} @ \mathrm{C}_{80}$ and act as a reservoir for angular momentum in the flip of the magnetization.

\section{CONCLUSIONS}

In conclusion $\mathrm{x}$-ray magnetic circular dichroism establishes noncollinear magnetism of the Dy ions and quasicollinear magnetism of $\mathrm{Gd}$ in $\mathrm{Dy}_{2} \mathrm{GdN} @ \mathrm{C}_{80}$. The groundstate energy difference between the ferromagnetic and the antiferromagnetic Dy configurations decreases relative to $\mathrm{Dy}_{2} \mathrm{ScN} @ \mathrm{C}_{80}$. From temperature-dependent magnetization decay measurements we, however, infer a higher kinetic barrier. The decrease in the prefactor for the description of the magnetic lifetime overcompensates the higher barrier and identifies Gd to catalize the decay of the magnetization in a single molecule magnet.

\section{ACKNOWLEDGMENTS}

We thank A. Seitsonen for the artwork in Fig. 1 and acknowledge financial support from the Swiss National Science Foundation (SNF Projects No. 200021L_147201, No. 206021_150784, No. 200020_153312, No. 200021_129861, No. 200021_147143, and No. PZ00P2-142474), the Deutsche Forschungsgemeinschaft (DFG Projects No. PO 1602/4-1 and No. 1602/5-1), and the Swedish Research Council (Grant No. 2015-00455).
[1] R. Sessoli, D. Gatteschi, A. Caneschi, and M. A. Novak, Magnetic bistability in a metal-ion cluster, Nature (London) $\mathbf{3 6 5}$, 141 (1993).
[2] D. Gatteschi, R. Sessoli, and J. Villain, Molecular Nanomagnets (Oxford University Press, Oxford, 2006). 
[3] E. Coronado, Molecular magnetism: from chemical design to spin control in molecules, materials and devices, Nat. Rev. Mater. 5, 87 (2020).

[4] N. Ishikawa, M. Sugita, T. Ishikawa, S. Koshihara, and Y. Kaizu, Lanthanide double-decker complexes functioning as magnets at the single-molecular level, J. Am. Chem. Soc. 125, 8694 (2003).

[5] D. N. Woodruff, R. E. P. Winpenny, and R. A. Layfield, Lanthanide single-molecule magnets, Chem. Rev. 113, 5110 (2013).

[6] S. T. Liddle and J. van Slageren, Improving f-element single molecule magnets, Chem. Soc. Rev. 44, 6655 (2015).

[7] J.-L. Liu, Y.-C. Chen, and M.-L. Tong, Symmetry strategies for high performance lanthanide-based single-molecule magnets, Chem. Soc. Rev. 47, 2431 (2018).

[8] C. A. P. Goodwin, F. Ortu, D. Reta, N. F. Chilton, and D. P. Mills, Molecular magnetic hysteresis at 60 kelvin in dysprosocenium, Nature (London) 548, 439 (2017).

[9] F.-S. Guo, B. M. Day, Y.-C. Chen, M.-L. Tong, A. Mansikkamaki, and R. A. Layfield, Magnetic hysteresis up to 80 kelvin in a dysprosium metallocene single-molecule magnet, Science 362, 1400 (2018).

[10] S. Stevenson, G. Rice, T. Glass, K. Harich, F. Cromer, M. R. Jordan, J. Craft, E. Hadju, R. Bible, M. M. Olmstead, K. Maitra, A. J. Fisher, A. L. Balch, and H. C. Dorn, Small-bandgap endohedral metallofullerenes in high yield and purity, Nature (London) 401, 55 (1999).

[11] A. A. Popov, S. Yang, and L. Dunsch, Endohedral fullerenes, Chem. Rev. 113, 5989 (2013).

[12] H. Funasaka, K. Sakurai, Y. Oda, K. Yamamoto, and T. Takahashi, Magnetic properties of $\mathrm{Gd} @ \mathrm{C}_{82}$ metallofullerene, Chem. Phys. Lett. 232, 273 (1995).

[13] R. Westerström, J. Dreiser, C. Piamonteze, M. Muntwiler, S. Weyeneth, H. Brune, S. Rusponi, F. Nolting, A. Popov, S. Yang, L. Dunsch, and T. Greber, An endohedral single-molecule magnet with long relaxation times: $\mathrm{DySc}_{2} \mathrm{~N} @ \mathrm{C}_{80}$, J. Am. Chem. Soc. 134, 9840 (2012).

[14] K. Junghans, C. Schlesier, A. Kostanyan, N. A. Samoylova, Q. Deng, M. Rosenkranz, S. Schiemenz, R. Westerstroem, T. Greber, B. Buechner, and A. A. Popov, Methane as a selectivity booster in the arc-discharge synthesis of endohedral fullerenes: Selective synthesis of the single-molecule magnet $\mathrm{Dy}_{2} \mathrm{TiC} @ \mathrm{C}_{80}$ and its congener $\mathrm{Dy}_{2} \mathrm{TiC}_{2} @ \mathrm{C}_{80}$, Angew. Chem., Int. Ed. 54, 13411 (2015).

[15] C. Schlesier, F. Liu, V. Dubrovin, L. Spree, B. Buechner, S. M. Avdoshenko, and A. A. Popov, Mixed dysprosium-lanthanide nitride clusterfullerenes $\mathrm{DyM}_{2} \mathrm{~N} @ \mathrm{C}_{80}-\mathrm{I}_{h}$ and $\mathrm{Dy}_{2} \mathrm{MN} @ \mathrm{C}_{80}-\mathrm{I}_{h}$ $(\mathrm{M}=\mathrm{Gd}, \mathrm{Er}, \mathrm{Tm}$, and $\mathrm{Lu})$ : synthesis, molecular structure, and quantum motion of the endohedral nitrogen atom, Nanoscale 11, 13139 (2019).

[16] L. Spree, C. Schlesier, A. Kostanyan, R. Westerstroem, T. Greber, B. Buechner, S. M. Avdoshenko, and A. A. Popov, Single-molecule magnets $\mathrm{DyM}_{2} \mathrm{~N} @ \mathrm{C}_{80}$ and $\mathrm{Dy}_{2} \mathrm{MN} @_{80}$ $(\mathrm{M}=\mathrm{Sc}, \mathrm{Lu})$ : The impact of diamagnetic metals on $\mathrm{Dy}^{3+}$ magnetic anisotropy, Dy $\cdots$ Dy coupling, and mixing of molecular and lattice vibrations, Chem. Eur. J. 26, 2436 (2020).

[17] J. D. Rinehart, M. Fang, W. J. Evans, and J. R. Long, Strong exchange and magnetic blocking in $\mathrm{N}_{2}^{3-}$-radical-bridged lanthanide complexes, Nat. Chem. 3, 538 (2011).
[18] L. Spree and A. A. Popov, Recent advances in single molecule magnetism of dysprosium-metallofullerenes, Dalton Trans. 48, 2861 (2019).

[19] R. Westerström, J. Dreiser, C. Piamonteze, M. Muntwiler, S. Weyeneth, K. Krämer, S.-X. Liu, S. Decurtins, A. Popov, S. Yang, L. Dunsch, and T. Greber, Tunneling, remanence, and frustration in dysprosium-based endohedral single-molecule magnets, Phys. Rev. B 89, 060406(R) (2014).

[20] M. Nie, J. Xiong, C. Zhao, H. Meng, K. Zhang, Y. Han, J. Li, B. Wang, L. Feng, C. Wang, and T. Wang, Luminescent singlemolecule magnet of metallofullerene DyErScN@ $\mathrm{I}_{h}-\mathrm{C}_{80}$, Nano Res. 12, 1727 (2019).

[21] G. van der Laan and A. I. Figueroa, X-ray magnetic circular dichroism-a versatile tool to study magnetism, Coord. Chem. Rev. 277-278, 95 (2014).

[22] J. Dreiser, K. S. Pedersen, C. Piamonteze, S. Rusponi, Z. Salman, Md. E. Ali, M. Schau-Magnussen, C. Aa. Thuesen, S. Piligkos, H. Weihe, H. Mutka, O. Waldmann, P. Oppeneer, J. Bendix, F. Nolting, and H. Brune, Direct observation of a ferri-to-ferromagnetic transition in a fluoride-bridged 3d-4f molecular cluster, Chem. Sci. 3, 1024 (2012).

[23] V. Corradini, A. Ghirri, E. Garlatti, R. Biagi, V. De Renzi, U. del Pennino, V. Bellini, S. Carretta, P. Santini, G. Timco, R. E. P. Winpenny, and M. Affronte, Magnetic anisotropy of $\mathrm{Cr}_{7} \mathrm{Ni}$ spin clusters on surfaces, Adv. Funct. Mater. 22, 3706 (2012).

[24] L. Badía-Romano, F. Bartolomé, J. Bartolomé, J. Luzón, D. Prodius, C. Turta, V. Mereacre, F. Wilhelm, and A. Rogalev, Field-induced internal $\mathrm{Fe}$ and $\mathrm{Ln}$ spin reorientation in butterfly $\mathrm{Fe}_{3} \mathrm{LnO}_{2}$ ( $\mathrm{Ln}=\mathrm{Dy}$ and Gd) single-molecule magnets, Phys. Rev. B 87, 184403 (2013).

[25] See Supplemental Material at https://link.aps.org/ supplemental/10.1103/PhysRevB.103.014404 for preparation of $\mathrm{Dy}_{2} \mathrm{GdN} @ \mathrm{C}_{80}$; Sum rule analysis of the XMCD data; Comparison of XMCD and SQUID data; Pseudospin model for the element specific magnetisation curves; Simulation results; $\chi^{2}$ maps and minima analysis; Determination of the Hysteresis area; Determination of the remanence time $T_{0}$ and comparison to previous evaluations; Reference $T_{0}$ measurements for $\mathrm{Dy}_{3} \mathrm{~N} @ \mathrm{C}_{80}$.

[26] C. Piamonteze, U. Flechsig, S. Rusponi, J. Dreiser, J. Heidler, M. Schmidt, R. Wetter, M. Calvi, T. Schmidt, H. Pruchova, J. Krempasky, C. Quitmann, H. Brune, and F. Nolting, X-Treme beamline at SLS: X-ray magnetic circular and linear dichroism at high field and low temperature, J. Synchrotron Radiat. 19, 661 (2012).

[27] S.-D. Jiang, B.-W. Wang, H.-L. Sun, Z.-M. Wang, and S. Gao, An organometallic single-ion magnet, J. Am. Chem. Soc. 133, 4730 (2011).

[28] Y. Teramura, A. Tanaka, B. T. Thole, and T. Jo, Effect of coulomb interaction on the $\mathrm{x}$-ray magnetic circular dichroism spin sum rule in rare earths, J. Phys. Soc. Jpn. 65, 3056 (1996).

[29] B. T. Thole, P. Carra, F. Sette, and G. van der Laan, X-Ray Circular Dichroism As a Probe of Orbital Magnetization, Phys. Rev. Lett. 68, 1943 (1992).

[30] P. Carra, B. T. Thole, M. Altarelli, and X. Wang, X-Ray Circular Dichroism and Local Magnetic Fields, Phys. Rev. Lett. 70, 694 (1993). 
[31] C. De Nadai, A. Mirone, S. S. Dhesi, P. Bencok, N. B. Brookes, I. Marenne, P. Rudolf, N. Tagmatarchis, H. Shinohara, and T. J. S. Dennis, Local magnetism in rare-earth metals encapsulated in fullerenes, Phys. Rev. B 69, 184421 (2004).
[32] A. Kostanyan, R. Westerstrom, D. Kunhardt, B. Buchner, A. A. Popov, and T. Greber, Sub-Kelvin hysteresis of the dilanthanide single-molecule magnet $\mathrm{Tb}_{2} \mathrm{ScN} @ \mathrm{C}_{80}$, Phys. Rev. B 101, 134429 (2020). 